\title{
Hacia un estudio bidimensional del Síndrome de Burnout en estudiantes universitarios
}

\author{
Towards a two-dimensional study of burnout syndrome \\ in college students
}

Yury Rosales Ricardo ${ }^{1}$

Fredy Rafael Rosales Paneque ${ }^{1}$

\footnotetext{
${ }^{1}$ Departamento de Cultura Física, Universidad Ciencias Médicas de Holguín. Independencia 91 entre Cuba y Prado, Reparto Vista Alegre. Holguín Cuba.

Abstract A literature review was conducted with a view to propose a new definition for burnout syndrome (BS) among university students, as a two-dimensional syndrome, where the two essential or core dimensions of BS are student exhaustion and student apathy and disinterest. It eliminates the third dimension of efficacy or academic self-efficacy in the specific case of students for not meeting the situational and conceptual requirements of the syndrome per se.

Key words Burnout syndrome, Two-dimensionality, Students
Resumen Se realizó un estudio de revisión con el objetivo proponer una nueva definición del Sindrome de Burnout (SB) en estudiantes universitarios, como un sindrome bidimensional, donde las dos dimensiones imprescindibles o corazón del SB estudiantil son el agotamiento y el desinterés estudiantil. Se elimina la tercera dimensión eficacia o autoeficacia académica en el caso particular de los estudiantes por no cumplir los requisitos situacionales y conceptuales del sindrome en sí.

Palabras clave Síndrome de Burnout, Bidimensionalidad, Estudiantes 


\section{Introducción}

El Síndrome de Burnout (SB), también conocido como síndrome del desgaste o agotamiento físico y mental, constituye un problema de gran repercusión social en nuestros días. El interés que en la actualidad despierta el Síndrome de Burnout ha facilitado una ampliación de su campo de estudio, pues se empezaron investigaciones en otros ámbitos profesionales y mucho más recientemente surgen los estudios con estudiantes universitarios.

Muchos estudiantes logran desarrollar las competencias necesarias en su proceso formativo que favorecen la consecución de sus objetivos académicos, mientras que otros jóvenes presentan dificultades que sólo se registran en indicadores de elevada mortalidad académica En esta última situación, los estudiantes generalmente experimentan una alta carga de desgaste mental y físico durante su proceso formativo.

Algunos estudiantes logran desarrollar estrategias adecuadas para afrontar las exigencias académicas, mientras que otros no lo hacen, y llegan a sentirse impedidos para modificar la situación problemática, lo cual trae como consecuencia el uso de comportamientos de escape o evitación como formas de afrontamiento que no son necesariamente las apropiadas en esta situación. Como se sabe, los problemas que no se resuelven, crecen y se acompañan de un proceso acumulativo de malestar prolongado ${ }^{1}$.

En estudiantes universitarios es una línea de investigación novedosa y muy reciente. Los estudios realizados en este sentido han estado dirigidos a la confirmación de la incidencia del síndrome en estas muestras pre profesionales.

Las instituciones de educación superior suelen ser lugares altamente estresantes; en dichas instituciones los alumnos se enfrentan de manera cotidiana a una serie de demandas que bajo su propia valoración se constituyen en situaciones generadoras de estrés, estas situaciones cuando son prolongadas y frecuentes pueden conducir al Síndrome de Burnout ${ }^{2}$.

En relación con otros tipos de estudiantes, en el universitario estas situaciones se pueden incrementar pues los programas de estudios de la mayoría de las carreras están considerablemente cargados en volumen de contenido, horas clase, mucho tiempo de auto estudio y realización de trabajos independientes además del proceso de creación de conocimiento y retroalimentación importante que ocurre en este nivel de enseñanza. Todo esto puede combinarse con los problemas personales, propios de cada estudiante.
Sin embargo los instrumentos para diagnosticar el Síndrome en estudiantes no han sido aplicados y validados para nuestra población universitaria cubana. Incluso no siquiera se han realizado estudios exploratorios que determinen esta entidad tan dañina para la salud mental y física de nuestros estudiantes.

Hay que decir que todo esto parte de un insuficiente estudio integrador del Síndrome de Burnout que se produce en los estudiantes universitarios cubanos que limita las posibilidades de diagnóstico del mismo y por tanto su estabilidad psicosomática, partiendo de las deficiencias de los instrumentos de diagnóstico utilizados hasta hoy y el enfoque de estos.

Como se ha podido observar, hasta el momento el Síndrome de Burnout ha sido estudiado solo como constructo tridimensional o unidimensional, primando sustantivamente las investigaciones en la primera. Es a partir de la definición utilizada que se aplican los instrumentos elaborados tridimensionales o unidimensionales.

Tridimensionalmente las dimensiones utilizadas para su estudio en trabajadores han sido el agotamiento emocional y la despersonalización, consideradas corazón del Síndrome y una tercera que ha sido criticada en varios estudios por su tal vez poca implicación en la formación teórica de éste, definida como realización personal.

En el caso de estudiantes cuando se ha estudiado como un síndrome tridimensional las tres dimensiones han sido el agotamiento emocional, el cinismo y la eficacia o autoeficacia académica, siendo criticada esta última de una manera similar al caso de los trabajadores.

A través de estudios se ha puesto de manifiesto que las dimensiones centrales del Burnout, específicamente en estudiantes, son el agotamiento y el cinismo. Por otra parte el "tercer componente" del Burnout (eficacia), se ha criticado en muchos sentidos. Por ejemplo, algunos autores señalan que: "la eficacia es un constructo cercano a una dimensión más estable de la personalidad y no tanto un componente central del Burnout"3.

Los resultados de un meta-análisis reciente confirman que: "El papel independiente de la eficacia de las otras dos dimensiones del Burnout con una correlación menor y en algunos casos no significativa"4.

Es por tanto el objetivo de este trabajo el proponer una nueva definición del Síndrome de Burnout, específicamente en estudiantes universitarios. Pues la mayoría de los estudios demuestran la implicación de las dimensiones agotamiento y cinismo como parte imprescindible del 
síndrome en estudiantes. Pero sin definir aún esta nueva visión y estudio, pues sólo se menciona.

\section{Discusión}

\section{Enfoque e Instrumentos para el diagnóstico del Síndrome de Burnout (SB) en estudiantes}

Entre las poblaciones identificadas como de riesgo para el desarrollo del SB se puede encontrar la universidad estudiantes ${ }^{5-10}$. Balogun et al. ${ }^{11}$ argumentan que el entorno competitivo encontró entre estudiantes, maestros y supervisores, conduce a conflictos que pueden conducir al estrés emocional y agotamiento. Otro problema señalado es la falta de tiempo para el ocio, la familia, amigos y necesidades personales, así como preocupaciones sobre el futuro profesional.

Es a partir del enfoque de estudio con el que se defina el SB que se utilizan los instrumentos elaborados para dicho fin. Sea el enfoque tridimensional, que ha sido por mucho el más utilizado, o el unidimensional.

El desarrollo del estudio del SB ha sido posibles gracias, entre otros aspectos, al desarrollo de instrumentos de diagnóstico validados. La investigación sobre SB ha señalado que existe un lenguaje común (en términos de medida) que viene del Maslach Burnout Inventory (MBI) que ha sido (y es) la medida dominante del SB en su enfoque y definición tridimensional. Realmente, es a partir de la publicación en el año 1981 del Maslach Burnout Inventory (MBI) por Maslach y Jackson cuando se establecen unos criterios de medida y evaluación del burnout, y se extiende el uso de un instrumento de medida estandarizado.

No obstante, desde esta primera época hasta la actualidad, el estudio del SB ha pasado por distintas etapas.

De forma originaria el MBI se construyó de manera exclusiva para medir burnout en educadores y personal del sector servicios (personal de enfermería, asistentes sociales, etc.). Estamos hablando del Maslach Burnout Inventory-Human Services Survey (MBI-HSS). Este instrumento evaluaba el burnout mediante tres dimensiones: agotamiento emocional, despersonalización y reducida realización personal. Debido a que el cuestionario podía aplicarse de una forma rápida y sencilla ha sido muy utilizado, facilitando así que la definición de burnout implícita en el MBI se convirtiese en la más aceptada. El Maslach Burnout Inventory (MBI) de Maslach y Jackson ha sido ampliamente utilizado, hasta el punto de que el resto de los instrumentos apenas han tenido un desarrollo significativo en la literatura científica ${ }^{11}$.

Existen, no obstante, otras medidas alternativas de burnout, En los pocos estudios unidimensionales encontrados es el agotamiento la única dimensión que se estudia. Los instrumentos más utilizados según esta definición son el Burnout Measure $(\mathrm{BM})^{12}$, El Copenhaguen Burnout Inventory $(\mathrm{CBI})^{13}$ y su versión para estudiantes en portugués $(\mathrm{CBI}-\mathrm{SS})^{8}$, La Escala de Cansancio Emocional (ECE) ${ }^{14}$ y la Escala Unidimensional de Burnout Estudiantil (EUBE) ${ }^{15}$. Todas definen el Síndrome como un estado de agotamiento físico, emocional y cognitivo producido por el involucramiento prolongado en situaciones generadoras de estrés ${ }^{16-19}$.

El Oldenburg Burnout Inventory $(\mathrm{OLBI})^{20} \mathrm{y}$ su versión para estudiantes en portugués ${ }^{7}$ están basados en la argumentación de que existen limitaciones psicométricas en el MBI debido a que los ítems en las mismas escalas van en la misma dirección (en positivo o en negativo). El OLBI fue elaborado para mitigar estos posibles sesgos del MBI. Está basado en una conceptualización similar al $\mathrm{MBI}^{20-22}$.

El Burnout Measure ${ }^{12}$ se diseñó alrededor de una medida simple de Síndrome de Burnout: el agotamiento, es por tanto unidimensional. Los ítems están escritos en términos generales y por tanto se pueden aplicar a cualquier grupo ocupacional. No obstante, la investigación sobre esta medida ha identificado un número de problemas resultantes de su estructura factorial y sus bases teóricas subyacentes, y se ha utilizado también sólo en el ámbito laboral ${ }^{23}$.

Resulta especialmente interesante resaltar que aunque la definición de Síndrome de Burnout que ofrecen Pines et al. ${ }^{24}$ ha sido, junto a la de Maslach y Jackson ${ }^{25}$, la más aceptada para comprender el síndrome, no ha sucedido lo mismo con los instrumentos de medida derivados. De hecho, mientras el MBI, como ya comprobamos, ha sido y es objeto de una amplia línea de investigación, el BM se ha sometido a contraste en pocas ocasiones, a pesar de que los resultados existentes indican unas características psicométricas apropiadas. Quizás su déficit pueda estar en la conceptualización misma de lo que pretenden medir: ¿es tedium o burnout?, o más importante ¿es el tedium un constructo similar al burnout? Con todo, mientras el conocimiento que se tiene del MBI es amplio, el desarrollo de una verdadera línea de investigación sobre el BM está 
por realizarse, a pesar de crearse ambas escalas en el mismo tiempo ${ }^{26}$.

Finalmente, el Copenhaguen Burnout Inventory (CBI) permite evaluar el burnout libre de contexto y es unidimensional, pues fue formulado considerando el agotamiento como constructo central de SB. Consiste en 19 ítems-preguntas divididas en tres elementos del SB: SB persona, SB en el trabajo, SB hacia los clientes ${ }^{13}$.

Otros investigadores han desarrollado una escala de agotamiento alternativa denominada 'cansancio cognitivo' (cognitive weariness) que incluye ítems como "Tengo problemas de concentración" y "Estoy ausente"27.

A pesar de las múltiples medidas de evaluación del burnout, puede decirse que el MBI sigue siendo el instrumento por excelencia para la medida y evaluación del burnout. Es más, se ha llegado a construir un instrumento de medida genérico que es útil para medir burnout en todo tipo de trabajos independientemente de las tareas que en él se realicen ${ }^{28}$. Este es el llamado MBI-GS (Maslach Burnout Inventory-General Survey) publicado en el año 1996 por Schaufeli et al. ${ }^{29}$, siendo un instrumento basado en este original y aplicable a todas las ocupaciones y trabajos ${ }^{30,31}$.

Es más, la extensión del SB no ha finalizado con su detección y medida en profesionales fuera del área de los servicios y del área de trabajos con datos y con objetos, sino que también se ha llegado a detectar en estudiantes universitarios diseñándose medidas específicas para su evaluación. La publicación del MBI-SS (Maslach Burnout Inventory-Student Survey) por Schaufeli et al. ${ }^{32}$ ha hecho posible medir el burnout fuera del ámbito ocupacional al definir sus dimensiones en referencia al estudio de este en estudiantes ${ }^{29}$.

El SB es una enfermedad crónica caracterizada por un estado de agotamiento total de la energía individual, asociado a una frustración intensa con actividades de trabajo ${ }^{33}$. El instrumento más comúnmente utilizado para identificar el síndrome de burnout es el Maslach Burnout Inventory $(\mathrm{MBI})^{31}$, que actualmente cuenta con cuatro versiones diferentes dirigidas a: Profesionales de la población general (MBI-GS), con participación humana grande (MBI-HSS), los profesores (MBI-ES) y los estudiantes (MBI-SS) ${ }^{34}$. Aunque diversas versiones del MBI han sido propuesta con el fin de mejorar la detección de Burnout en diferentes grupos de población, varios autores han señalado las fallas teóricas y metodológicas en el diseño de el MBI, que puede poner en peligro los resultados cuando el instrumento se utiliza en diferentes poblaciones de la que se desarrolló originalmente $e^{13,20,35,36}$.
A pesar de la evidente necesidad de estudios sobre el SB en los estudiantes, estos son incipientes según Maroco y Tecedeiro ${ }^{37}$ y Maroco et al..$^{38}$, que puede estar ocurriendo debido a la falta de adecuada instrumentos para evaluar este síndrome en los estudiantes. Prácticamente el único instrumento propuesto en la literatura para evaluar el síndrome en los estudiantes es el Maslach Burnout Inventory de Estudiantes (MBISS $)^{39}$, cuyo propiedades psicométricas han sido evaluados y su estabilidad atestiguado en varios estudios, incluido el universitario estudiantes en Portugal $^{37,39}$ y Brasil $^{40}$.

Sin embargo, el MBI ha sido el blanco de las críticas por varios autores ${ }^{13,21}$. Demerouti et al..$^{20,21}$ y Halbesleben y Demerouti ${ }^{41}$ critican el hecho de que la dimensión Agotamiento y Cinismo MBI tienen una formulación positiva, mientras que la eficacia profesional dimensión tiene formulación negativa, que se compromete la sensibilidad del inventario y su discriminatoria. Otro aspecto destacado por los autores es que el MBI sólo tiene en cuenta los aspectos emocionales de la dimensión agotamiento excluyendo los elementos físi$\cos$ y cognitivos ${ }^{42-44}$.

Teniendo en cuenta las críticas que hizo en relación con el MBI, Demerouti y Nachreiner ${ }^{45}$ propusieron el Inventario Oldenburg de Burnout (OLBI), que consta de 16 ítems que describen diferentes estados de agotamiento emocional, desapego y de acuerdo con cada elemento en escala ordinal de 4 puntos (de 1 - totalmente en desacuerdo a 4 Totalmente de acuerdo). La mitad de los artículos se formularon positivamente y medio fueron formulados negativamente. Este instrumento se basa en un modelo teórico similar al de Maslach. La validez de OLBI se puso a prueba en diferentes grupos de población $\mathrm{n}^{20-22,46-48}$.

Tomando en consideración que OLBI fue desarrollado para satisfacer los diferentes grupos profesionales, su uso en los estudiantes puede ser una alternativa interesante para MBI-SS aunque no fue diseñado con ese objetivo. Aunque se considera que la dimensión desapego utilizada por el instrumento no es la más correcta para su uso en estudiantes en nuestro contexto iberoamericano y específicamente hispanohablante.

Robinson y Merry ${ }^{49}$ ponen de manifiesto las dificultades de usando el MBI en poblaciones que no tienen un importante participación humana en sus actividades de trabajo o que pertenecen a otras culturas. Doty e Glick ${ }^{50}$ y Milfont et al. ${ }^{51}$ critican el hecho de que las tres subescalas, propuso en el instrumento, no se dispone de los elementos formulados en la misma dirección, que también fue señalado por Demerouti et al.. ${ }^{20,21}$. Estos auto- 
res también agregan como limitaciones de la MBI el hecho de que la dimensión de Agotamiento. Considera los aspectos emocionales, sin incluir la física y los aspectos cognitivos. Kristensen et al. ${ }^{13}$ y Yeh et al. ${ }^{52}$ consideran que el agotamiento es el aspecto central del síndrome.

De esta manera, Carlotto y Câmara ${ }^{40}$, Dyrbye et al. ${ }^{53}$, Martínez et al. ${ }^{54}$ y Salanova et al..$^{55}$ hacen hincapié en que la detección temprana de importantes niveles sintomáticos de SB puede ser un indicador de posibles problemas en la escuela, permitiendo intervenciones preventivas. Maroco y Tecedeiro ${ }^{37}$ informaron que los estudiantes cuando se enfrentan a presiones sociales y profesionales, que expresen sobre el financiamiento de los estudios y las relaciones con son altamente propensos a desarrollar los profesores y compañeros, SB. La aparición de este síndrome puede seriamente limitar tanto el bienestar psicosocial y lo académico rendimiento de estos estudiantes. Por lo tanto, para la detección de agotamiento es necesario el uso de medición fiable y válido instrumentos que permitan un diagnóstico fiable y válido $^{56,57}$.

Lamentablemente, no existe, en estos instrumentos, un criterio unánime entre los expertos para establecer el diagnóstico, ni sobre los porcentajes de prevalencia e incidencia, pues los criterios varían para cada estudio ${ }^{58}$.

Por esta ausencia de criterios claros, muchos estudios han recurrido a la determinación del síndrome utilizando criterios estadísticos ligados a la muestra: el uso de una o media desviación estándar alrededor de la media, o el uso de un número de percentiles predeterminados (el tercil o el cuartil superior, son los más frecuentes), lo cual sesga la aparición del síndrome en la muestra sin que esto sea surge la necesidad de establecer indicadores que ayuden mediante criterios objetivos y subjetivos, y establecer los puntos de corte de los instrumentos que determinan la presencia del síndrome.

En el 2008 el Dr. Arturo Barraza ${ }^{15}$ elaboró y validó la Escala Unidimensional de Burnout Estudiantil. Esta consta de 15 ítems mide nivel de agotamiento físico, emocional y cognitivo en los estudiantes, respetando el concepto unidimensional del Síndrome de Burnout y reportó un nivel de confiabilidad de 91 en alfa de Cronbach y de 90 en la confiabilidad por mitades según la fórmula de Spearman-Brown ${ }^{15}$.

Lo anteriormente expuesto, muestra la necesidad de realizar un estudio de corte longitudinal en contextos académicos donde se evalúe el síndrome en estudiantes desde una mirada clínica mediante instrumentos elaborados y validados en Latinoamérica, y utilizando técnicas cualitativas, y un enfoque y definición más correctas del SB en estudiantes. De esta manera, se podrá inicialmente establecer la prevalencia e incidencia del síndrome y realizar a partir de éste una mejor prevención e intervención.

\section{Enfoques y dimensiones}

A través de estudios se ha puesto de manifiesto que las dimensiones centrales del SB son el agotamiento y el cinismo. Por otra parte el tercer componente del SB (eficacia) se ha criticado en muchos sentidos. Algunos autores señalan que: la eficacia es un constructo cercano a una dimensión más estable de la personalidad y no tanto un componente central del SB ${ }^{48}$.

En 1997 Maslach \& Leiter publicaron el libro "The Truth About Burnout" en el que proponen una reconceptualización del SB como una "crisis general" en la relación de la persona con su trabajo. Crisis que se ha venido definiendo en función de tres dimensiones más genéricas que las anteriormente propuestas y que no se refieren exclusivamente al trabajo de ayuda a personas sino que son aplicables a cualquier situación. Estas dimensiones son: agotamiento emocional y también físico; actitud cínica de distanciamiento mental hacia el trabajo y sentimientos de inadecuación profesional y de pérdida de confianza en las propias capacidades profesionales. De estas tres dimensiones las dos primeras, agotamiento y distanciamiento (entendida como despersonalización o cinismo) se han considerado las dimensiones centrales o "corazón del burnout". El tercer componente del SB - eficacia - se ha criticado su rol de "dimensión" del SB ya que parece desempeñar un rol distinto. Los resultados de un meta-análisis reciente confirman el papel independiente de la eficacia profesional. Con una correlación menor y en algunos casos no significativa ${ }^{4}$.

No hay razón para suponer que el SB se limita a los servicios humanos. La estructura básica de $\mathrm{SB}$ es el mismo entre las ocupaciones, es decir, la combinación de agotamiento y distanciamiento. En los servicios humanos estas dimensiones están relacionadas con el trabajo con la gente, ya que constituyen el objeto de trabajo del empleado, y se manifiestan en el agotamiento como resultado de tensión interpersonal (agotamiento emocional) y el distanciamiento (despersonalización o cinismo).

Por tanto el concepto de SB se puede aplicar al trabajo como a actividades fuera del contexto del trabajo que se estructuran, coercitivo en 
la naturaleza, y dirigida hacia objetivos específicos. En otras palabras, a las actividades que, desde una perspectiva psicológica, son similares al trabajo. Estas actividades no sólo pueden ser agotadoras, pero también pueden permitir a las personas a distanciarse de ella. Un ejemplo es el SB en estudiantes. Aunque los estudiantes no son ni empleados ni ejercen puestos de trabajo, desde una perspectiva psicológica sus actividades principales se pueden considerar «trabajo». Ellos asisten a clases y hacen las tareas (Actividades coercitivas estructurados) con el fin de aprobar los exámenes y adquirir un título (objetivos específicos $)^{59}$.

En un análisis la Dra. Shaufeli y Taris expresan si son tres dimensiones demasiados para el estudio y definición del Síndrome. El SB consiste en al menos dos dimensiones (la fatiga y el distanciamiento, tal vez suplementado con falta de eficacia) que puede medirse general (es decir, el uso de elementos aplicable a una amplia variedad de ocupaciones) o específicamente (con elementos adaptados a un tipo de única ocupación). Por lo tanto, el agotamiento puede manifestarse en los estudiantes por sentirse agotado por estudio demandas y tener una actitud cínica e indiferente hacia el estudio de uno ${ }^{30}$.

Kristensen et al. ${ }^{13}$ consideran que son tres dimensiones demasiados para la caracterización de $\mathrm{SB}$, y reducen el Síndrome a una sola dimensión tocando la fatiga física y mental y el cansancio. Esto ha sido sugerido antes y varias escalas de factor único se pueden encontrar en la literatura que disminuyen el SB a solamente la dimensión agotamiento (por ejemplo, Pines y Aronson ${ }^{12}$, Shirom ${ }^{60}$ ). Del mismo modo, la escala de agotamiento del MBI ha sido frecuentemente utilizada solo como una medida de 'burnout'. Pero ¿por qué equiparar el SB con agotamiento? Cuando el $\mathrm{SB}$ es idéntico al agotamiento, el término es redundante y no hay necesidad de desarrollar otro instrumento. En ese caso, se podrían utilizar los inventarios de agotamiento existentes ${ }^{13}$.

Kristensen et al..$^{13}$ son inclinados a creer que el SB se puede medir usando menos de tres dimensiones. El agotamiento y el distanciamiento (entendida como despersonalización o cinismo) constituyen dos partes inseparables del fenómeno del Burnout, son su estructura básica ${ }^{13}$.

Existe cada vez más una tendencia a centrar la aproximación conceptual del SB en el agotamiento ${ }^{4}$ y su secuela habitual de cinismo o distanciamiento, tal como tiende a ser actualmente denominado. Aunque son muchos otros los aspectos que pueden ser considerados, estos dos serían sus aspectos centrales. Desde esta perspectiva, el SB puede ser definido como "un estado de agotamiento físico, emocional y mental causado por la implicación a lo largo del tiempo en situaciones que son emocionalmente demandantes"4.

Como resultado de ello, no pocas investigaciones se han centrado en analizar los factores que explican fundamentalmente el agotamiento emocional, considerado como la dimensión inicial y critica en la explicación del proceso ${ }^{44}$. Igualmente, se ha encontrado evidencia a través de estudios longitudinales que el agotamiento emocional sería la dimensión que precede en el desarrollo de otros posibles aspectos o dimensiones de burnout ${ }^{61}$.

El tercer componente de Síndrome, la eficacia, puede o bien actuar como un precursor o como consecuencia del agotamiento, dependiendo de perspectiva. En el primer caso la eficacia actuaría como una falta personal recursos, mientras que en este último caso se trataría de una auto-evaluación.

Hay que hacer notar que los estudios recientes han sugerido que la dimensión de la realización personal (entendida también como eficacia o autoeficacia) no puede ser parte del concepto total de burnout: "Se hace cada vez más evidente a partir de estudios con el MBI original que personal logro desarrolla en gran medida independiente de las otras dos dimensiones del burnout"62. Hay un consenso amplio en considerar la dimensión "realización personal o eficacia" más como un factor independiente que como una dimensión interna del síndrome; sería más bien consecuencia del desgaste profesional ${ }^{63}$ o un modulador cercano al constructo de autoeficacia ${ }^{64}$.

Por tanto, luego de haber realizado la investigación utilizando una seria revisión de literatura nacional e internacional en bases de datos científicas sobre los estudios realizados en estudiantes, las definiciones con las que se ha trabajado y elaborado los instrumentos en esta población en particular, las dimensiones e ítems de estos, se propone una nueva definición de este Síndrome específicamente en estudiantes universitarios:

Sindrome bidimensional compuesto por agotamiento y desinterés que ocurre en el contexto escolar universitario y puede provocar graves daños en la salud física y mental de los estudiantes.

\section{Propuesta de definiciones de las dimensiones}

Dimensión Agotamiento estudiantil: estado de agotamiento general por las demandas de es- 
tudio en la universidad. El estudiante no puede dar en clases más de sí mismo.

Dimensión Desinterés estudiantil: Actitudes de desinterés frente a la actividad académica y dudas acerca de la importancia de los estudios universitarios que realiza.

Esta última dimensión toma como base las definiciones de las dimensiones distanciamiento, despersonalización, cinismo y desapego que hasta ahora han sido utilizadas indistintamente para definir fenómenos similares.

\section{Conclusiones}

El estudio bidimensional del Síndrome de Burnout en estudiantes universitarios pudiera ser una nueva línea de investigación para la búsqueda de alternativas e intervenciones que sean punto de partida para una mejor prevención y tratamiento de este complejo síndrome que afecta la población estudiantil universitaria en toda Iberoamérica y el mundo.

\section{Colaboradores}

Y Rosales Ricardo y FR Rosales Paneque han participado igualmente en cada una de las etapas de elaboración de este artículo. 


\section{Referências}

1. Caballero C, Hederich C, Palacios SJ. El Burnout Académico: delimitación del síndrome y factores asociados a su aparición. Rev Latinoamericana de Psicología 2010; 42(1):131-146.

2. Rosales Ricardo Y, Rosales Paneque FR. Burnout estudiantil universitario. Conceptualización y estudio. $\mathrm{Sa}$ lud Mental 2013; 36(4):337-345.

3. Cordes CL, Dougherty TW. Una revisión y una integración de la investigación sobre Burnout. Academia de examen de la gestión 1993; 18:621-656.

4. Lee RT, Ashforth BE. Un examen meta-analítico de la correlación de las tres dimensiones del Burnout. Diario de Psicología Aplicada 1996; 81:123-133.

5. Feliciano KVO, Kovacs MH, Sarinho SW. Burnout entre médicos da Saúde da Família: os desafios da transformação do trabalho. Cien Saude Colet 2011; 16(8):3373-3382.

6. Lima RAS, Souza AI, Galindo RH, Feliciano KVO. Vulnerabilidade ao burnout entre médicos de hospital público do Recife. Cien Saude Colet 2013; 18(4):10511058.

7. Campos JADB, Carlotto MS, Marôco J. Copenhagen Burnout Inventory - Student Version: Adaptation and Transcultural Validation for Portugal and Brazil. Psicologia: Reflexão e Crítica 2012; 26(1):87-97.

8. Campos JADB, Carlotto MS, Marôco J. Oldenburg Burnout Inventory - Student Version: Cultural Adaptation and Validation into Portuguese. Psicologia: Reflexão e Crítica 2012; 25(4):709-718.

9. Appels A. Estrés laboral, agotamiento y enfermedad. In: Buendía J, editor. Estrés laboral y salud. Madrid: Biblioteca Nueva; 2006.

10. Aranda C, Pando M, Velásquez I, Acosta M, Pérez M. "Síndrome de Burnout y factores psicosociales, en estudiantes de postgrado del Departamento de Salud Pública de la Universidad de Guadalajara, México". Rev Psiquiatría Facultad de Medicina Barna 2003; 30(4):193-199.

11. Balogun JA, Helgemoe S, Pellegrini E, Hoeberlein T. Test-retest reliability of a psychometric instrument designed to measure physical therapy students' burnout. Perceptual \& Motor Skills 1995; 81(2):667-672.

12. Pines A, Aronson E. Career burnout: Causes and cures. New York: Free Press; 1988.

13. Kristensen TS, Borritz M, Villadsen E, Christensen KB. The Copenhagen Burnout Inventory: A new tool for the assessment of burnout. Work and Stress 2005; 19(3):192-207.

14. Ramos F, Manga D, Moran C. Escala de Cansancio Emocional (ECE) para estudiantes universitarios: Propiedades psicométricas y asociación. Interpsiquis 2005; 7 feb. [acceso 2013 abril 22]. Disponíble en: http://www. psiquiatria.com/ansiedad/estres-62/escala-de-can sancio-emocional-ece-para-estudiantes-universitarios-propiedades-psicometricas-y-asociacion/

15. Barraza A. Burnout Estudiantil. Un enfoque Unidimensional. Revista Electrónica de Psicología Científica.com 2008; 16. [acceso 2013 mar 28]. Disponible en: http:// www.psicologiacientifica.com/burnout-estudiantil/

16. Barraza A, Carrasco R, Arreola MG. Burnout Estudiantil. Un estudio exploratorio. In: Barraza A, Gutiérrez D, Ceniceros DI, coordinadores. Alumnos y Profesores en Perspectiva. Durango: Universidad Pedagógica de Durango; 2009. p. 68-84.
17. Barraza A. Validación psicométrica de la Escala Unidimensional del Burnout Estudiantil. Revista Intercontinental de Psicología y Educación 2011; 13(2):53-76.

18. Borda M, Navarro E, Aun E, Berdejo H, Racedo K, Ruiz J. Síndrome de Burnout en estudiantes de internado del Hospital Universidad del Norte. Revista Salud Uninorte 2007; 23(1):43-51.

19. Bresó E, Salanova M, Schaufeli WB. In search of the 'third dimension' of burnout. Applied Psychology: An International Review 2007; 56(3):460-478.

20. Demerouti E, Bakker AB, Nachreiner F, Schaufeli WB. The job demands-resources model of burnout. J Appl Psychol 2001; 86(3):499-512.

21. Demerouti E, Bakker AB, Vardakou I, Kantas A. The convergent validity of two burnout instruments - A multitrait-multimethod analysis. European Journal of Psy-chological Assessment 2003; 19(1):12-23.

22. Demerouti E, Mostert K, Bakker AB. Burnout and work engagement: A thorough investigation of the independency of both constructs. J Occup Health Psychol 2010; 15(3):209-222.

23. Schaufeli W B, van Dierendonck D. The construct validity of two burnout measures. Journal of Organizational Behavior 1993; 14(7):631-647.

24. Pines AM, Aronson E, Kafry D Burnout: From tedium to personal growth. New York: Free Press; 1981.

25. Maslach C, Jackson SE. The measurement of experienced burnout. Journal of Occupational Behavior 1981; 2:99-113.

26. Pines A. "Burnout: An Existential Perspective". In: Schaufeli WB, Maslach C, Marek T, editors. Professional burnout: recent developments in theory and research. London: Taylor \& Francis; 1993. p. 35-51.

27. Van Horn JE, Taris TW, Schaufeli WB, Schreurs PJG. The structure of occupational wellbeing: A study among Dutch teachers. Journal of Occupational and Organizational Psychology 2004; 77(3):365-377.

28. Schaufeli WB, Taris TW. Commentary. The conceptualization and measurement of burnout: Common ground and worlds apart. Work and Stress 2005; 19: 356-362.

29. Schaufeli WB, Leiter MP, Maslach C, Jackson SE. Maslach Burnout Inventory - General Survey. In: Maslach C, Jackson SE, Leiter MP. The Maslach Burnout Sección Monográfica Inventory-Test Manual. $3^{\text {rd }}$ ed. Palo Alto: Consulting Psychologists Press; 2000.

30. Schaufeli WB, Taris TW. The conceptualization and measurement of burnout: common ground and worlds apart. Work and Stress 2010; 19:256-262.

31. Salanova M, Schaufeli WB, Llorens S, Peiró JM, Grau R. Desde el "burnout" al "engagement": ¿Una nueva perspectiva? Rev Psicología del Trabajo y de las Organizaciones 2000; 16(2):117-134.

32. Schaufeli WB, Salanova M, González-Romá V, Bakker $\mathrm{AB}$. The measurement of engagement and burnout: A confirmatory factor analytic approach. Journal of Happiness Studies 2002; 3:71-92.

33. Maslach C, Schaufeli WB, Leiter MP. Job burnout. Annual Review of Psychology 2001;52: 397-422.

34. Schaufeli WB, Leiter MP, Maslach C. Burnout: Thirty-five years of research and practice. Career Development International 2009; 14(3):204-220.

35. Garden A M. Depersonalization: a valid dimension of burnout? Human relations 1987; 40:545-560. 
36. Milfont TL, Denny S, Ameratunga S, Robinson E, Merry, S. Burnout and wellbeing: Testing the Copenhagen Burnout Inventory in New Zealand teachers. Social Indicators Research 2008; 89(1):169-177.

37. Marôco J, Tecedeiro M. Inventário de Burnout de Maslach para estudantes portugueses. Psicologia, Saúde \& Doenças 2009; 10(2):227-235.

38. Marôco J, Tecedeiro M, Martins P, Meireles A. Estrutura fatorial de segunda ordem da Escala de Burnout de Malasch para estudantes numa amostra portuguesa. Análise Psicológica 2008; 26(4):639-649.

39. Schaufeli WB, Martinez I, Marques Pinto A, Salanova $M, B a k k e r A B$. Burnout and engagement in university students: A cross national study. Journal of Cross-Cultural Psychology 2002; 33:464-481.

40. Carlotto MS, Câmara SG. Características psicométricas do Maslach Burnout Inventory - Student Survey (MBISS) em estudantes universitários brasileiros. PsicoUSF 2006; 11(2):167-173.

41. Halbesleben JRB, Demerouti E. The construct validity of an alternative measure of burnout: investigating the English translation of the Oldenburg Burnout Inventory. Work and Stress 2005; 19:208-220.

42. Gutiérrez D. El Síndrome de Burnout en alumnos de educación secundaria. Investigación Educativa Duranguense 2008; 5(10):26-35.

43. Gutiérrez D. Prevalencia del Síndrome de Burnout en estudiantes de nivel medio superior. Investigación Educativa Duranguense 2009; 5(11):18-23.

44. Maslach C, Jackson SE, Leiter MP. Maslach Burnout Inventory. $3^{\text {rd }}$ ed. Palo Alto: Consulting Psychologist Press; 1996.

45. Demerouti E, Nachreiner F. ZurSpezifität vonBurnout für Dienstleistungsberufe: Fakt oder Artefakt? [The specificity of burnout in human services: Fact orartifact?].Zeitschrift für Arbeitswissenschaft 1998; 52:82-89.

46. Demerouti E, Bakker A B, Nachreiner F, Schaufeli WB. A model of burnout and life satisfaction among nurses. J Adv Nurs 2000; 32:454-464

47. Halbesleben JRB, Demerouti E. The construct validity of an alternative measure of burnout: investigating the English translation of the Oldenburg burnout inventory. Work and Stress 2005; 19(3):208-220.

48. Peterson U, Demerouti E, Bergström G, Samuelsson M, Asberg M, Nygren A. Burnout and physical and mental health among Swedish healthcare workers. J Adv Nurs 2008; 62(1):84-95.

49. Milfont TL, Denny S, Ameratunga S, Robinson E, Merry S. Burnout and wellbeing:testing the Copenhagen burnout inventory in New Zealand teachers. Soc Indic Res 2008; 89:169-177.

50. Doty DH, Glick WH. Common methods bias: Does common methods variance really bias results? Organizational Research Methods 1998; 1(4):374-406.

51. Milfont TL, Denny S, Ameratunga S, Robinson E, Merry S. Burnout and wellbeing: Testing the Copenhagen Burnout Inventory in New Zealand teachers. Social Indicators Research 2008; 89(1):169-177.
52. Yeh WY, Cheng Y, Chen CJ, Hu PY, Kristensen TS Psychometric properties of the Chinese version of Copenhagen burnout inventory among employees in two companies in Taiwan. International Journal of Behavioral Medicine 2007; 14(3):126-133.

53. Dyrbye LN, Thomas MR, Power DV, Durning S, Moutier C, Massie FS Junior, Harper W, Eacker A, Szydlo DW, Sloan JA, Shanafelt TD. Burnout and serious thoughts of dropping out of medical school: A multiinstitutional study. Academic Medicine 2010; 85(1):94102.

54. Martinez IMM, Pinto AM, Silva AL. Burnout em estudantes do ensino superior. Rev. Portuguesa de Psicologia 2000; 35: 151-16755.

55. Salanova M, Schaufeli W, Martinez I, Breso E. How obstacles and facilitators predict academic perfor-mance: The mediating role of study burnout and engage-ment. Anxiety Stress and Coping 2010; 23(1): 53-70.

56. Carlotto MS, Câmara GS. Preditores da Síndrome de Burnout em estudantes universitários. Pensamiento Psicológico 2008; 4(10):101-109.

57. Carlotto MS, Tarnowski M. Síndrome de Burnout em estudantes de psicología. Temas em Psicología 2007; 15(2):173-180.

58. Moreno-Jiménez B, Carvajal RR, Escobar RE. "La evaluación del burnout profesional. Factorialización del MBI-GS. Un análisis preliminar. Ansiedad y Estrés 2001; 7(1):69-78.

59. Rosales Ricardo Y. Estudio unidimensional del Síndrome de Burnout en estudiantes de medicina de Holguín. Rev. Asoc. Esp. Neuropsic 2012; 32(116):521-545.

60. Shirom A. Job-related burnout: A review. In: Quick JC, Tetrick LE, editors. Handbook of Occupational Health Psychology. Washington, DC: American Psychological Association; 2003. p. 245-265.

61. Moreno-Jiménez B, Rodríguez-Carvajal R, Garrosa Hernández E, Morante Benadero MA. Terminal versus non-terminal care in physician burnout: the role of decision-making processes and attitudes to death. Salud Ment 2008; 31(2):93-101.

62. Schutte N, Toppinnen S, Kalimo R, Schaufeli WB The factorial validity of the Maslach Burnout Inventory-General Survey (MBI-GS) across ations and occupations. Journal of Occupational and Organizational Psychology 2000; 73:53-66.

63. Shirom A. Burnout in work organization. In: Cooper CL, Robertson I, editors. Internacional review of industrial and organizational psychology. Nueva York: John Wiley and Sons; 1989

64. Salanova Soria M, Martinez I, Breso E, LLorens G. Bienestar psicológico en estudiantes universitarios: facilitadores y obstaculizadores del desempeño académico. Anales de Psicología 2005; 21(1):170-180.

Artigo apresentado em 02/08/2013

Aprovado em 27/09/2013

Versão final apresentada em 02/10/2013 
